\title{
Tensor fasciae latae muscle in human embryos and foetuses with special reference to its contribution to the development of the iliotibial tract
}

\author{
K.H. Cho' ${ }^{1}$, Z.W. Jin², H. Abe ${ }^{3}$, J. Wilting ${ }^{4}$, G. Murakami ${ }^{5}$, J.F. Rodríguez-Vázquez ${ }^{6}$ \\ ${ }^{1}$ Department of Neurology, Wonkwang University School of Medicine and Hospital, Republic of Korea \\ ${ }^{2}$ Department of Anatomy, Wuxi Medical School, Jiangnan University, Wuxi, China \\ ${ }^{3}$ Department of Anatomy, Akita University School of Medicine, Japan \\ ${ }^{4}$ Department of Anatomy, School of Medicine, Georg-August-Universität Götingen, Germany \\ ${ }^{5}$ Division of Internal Medicine, Iwamizawa Asuka Hospital, Japan \\ ${ }^{6}$ Department of Anatomy and Human Embryology, Institute of Embryology, Faculty of Medicine, \\ Complutense University, Madrid, Spain
}

[Received: 1 November 2017; Accepted: 13 December 2017]

Background: The human tensor fasciae latae muscle (TFL) is inserted into the iliotibial tract and plays a critical role in lateral stabilisation of the hip joint. We previously described a candidate of the initial iliotibial tract that originated from the gluteus maximus muscle and extended distally.

Materials and methods: This study extended our observations by examining 30 human embryos and foetuses of gestational age (GA) 7-14 weeks (crown-to-rump length 24-108 mm). At GA 7 weeks, the TFL appeared as a small muscle mass floating in the subcutaneous tissue near the origins of the gluteus medius and rectus femoris muscles.

Results: Subsequently, the TFL obtained an iliac origin adjacent to the rectus femoris tendon, but the distal end remained a tiny fibrous mass on the vastus lateralis muscle. Until GA 10 weeks, the TFL muscle fibres were inserted into a vastus lateralis fascia that joined the quadriceps tendon distally. The next stage consisted of the TFL muscle belly "connecting" the vastus fascia and the gluteus fascia, including our previous candidate of the initial iliotibial tract. Until GA 14 weeks, the TFL was sandwiched by two laminae of the connecting fascia.

Conclusions: These findings suggested that, when the vastus lateralis fascia separated from the quadriceps tendon to attach to the tibia, possibly after birth, the resulting iliotibial tract would consist of a continuous longitudinal band from the gluteus maximus fascia, via the vastus fascia, to the tibia. Although it is a small muscle, the foetal TFL plays a critical role in the development of the iliotibial tract. (Folia Morphol 2018; 77, 4: 703-710)

Key words: tensor fasciae latae muscle, iliotibial tract, fascia lata, bipedal gait, human foetus 


\section{INTRODUCTION}

The tensor fasciae latae muscle (TFL) arises from the anterior part of the outer lip of the iliac crest. A thick band-like aponeurosis, the iliotibial tract or band, splits into two laminae to sandwich the TFL; the two laminae subsequently join together to meet the gluteal aponeurotic fascia and extend inferiorly to the Gerdy tubercle at the anterolateral tibia $[5,11]$. Together with the gluteus medius and minimus muscles, the TFL acts to balance the weight of the body and the non-weight-bearing leg during walking [9]. During the stance phase of walking, the TFL fixes the hip and femur together, whereas, while standing, the TFL counteracts body weight [1]. Electromyography showed that the anteromedial muscle fibres of the TFL had a greater mechanical advantage for hip flexion than the posterolateral fibres, whereas the latter have greater mechanical advantage for hip abduction and internal rotation [12]. The TFL also plays a distinct role in various exercises, such as kicking [3], as well as in pathologic states that require compensatory muscle actions under conditions of strain and pain [8]. The TFL is hypertrophied in patients with a tear of the gluteal tendon [15]. Thus, despite its small size, the TFL enhances the function of other muscles.

The TFL is thought to develop along with the gluteus medius muscle without any clear demarcation between them [4]. Our recent study of human foetuses reported a specific fascial structure originating from the gluteus maximus muscle, extending distally by a possible traction of the biceps femoris muscle and dispersing in the subcutaneous tissue [13]. Although originally considered a fascial extension, it was too short to reach the tibia. According to Shiraishi et al. [13], the initial TFL appeared to be floating in the subcutaneous tissue without any origin or insertion. To date, however, there is no information on the involvement of the TFL in the final iliotibial tract. Consequently, using histological sections from human embryos and foetuses at gestational age (GA) 7-14 weeks (crownrump length [CRL] 24-108 $\mathrm{mm}$ ), the aim of this study was to describe the developing TFL and iliotibial tract.

\section{MATERIALS AND METHODS}

The study was performed in accordance with the provisions of the Declaration of Helsinki 1995 (as revised in 2013). Serial sections of pelvises and lower extremities obtained from 30 human embryos and foetuses at GA 7-14 weeks were divided into two groups. The first consisted of 10 embryos of GA
7-8 weeks (CRL 24-35 mm) and the second of 20 foetuses of GA 10-14 weeks (CRL 53-108 mm). The sectional planes were sagittal, frontal or horizontal, but often tilted to make an intermediate plane between the frontal and horizontal planes. Eight of the 30 embryos and foetuses, including six of GA 7-8 weeks and two of GA 10-14 weeks, belonged to the Blechschmidt collection in the Medical Museum of Georg-August-Universität Göttingen. Serial sagittal sections of these foetuses had been prepared. Most sections were stained with haematoxylin and eosin (H\&E), although some were stained with azan or Masson trichrome stain. Use of samples from this collection did not require specific approval of the Institute. The other 22 embryos and foetuses (4 of GA 7-8 weeks and 18 of GA 10-14 weeks) were part of the large collection kept at the Embryology Institute of the Universidad Complutense, Madrid, and were the products of miscarriages and ectopic pregnancies at the Department of Obstetrics of the University. Most sections were stained with H\&E, while some were stained with azan, orange $G$ or silver stain. The study was approved by the Complutense University ethics committee (B08/374). Observations and taking photographs were usually performed with Nikon Eclipse 80, but photos at ultra-low magnification (objective lens less than $\times 2$ ) were taken using a high-grade flat scanner with translucent illumination (Epson scanner GTX970).

\section{RESULTS}

\section{Observations of smaller specimens}

At GA 7-8 weeks (Figs. 1, 2), a small muscle mass of the TFL was seen between the rectus femoris and gluteus medius muscles near the iliacus. This muscle appeared to be attached to the iliacus on the immediately lateral side of the origin of the rectus femoris (Fig. 1A, B), but its bony origin was unclear. Thus, the initial TFL appeared to be floating in subcutaneous tissue. Because the small muscle belly ran along the medial or anterior margin of the gluteus medius, the TFL appeared to be divided from the latter muscle (Fig. 2C, D). Muscle fibres of the TFL extended inferiorly or distally along the lateral surface of the vastus lateralis muscle and ended as a tiny fibrous mass on the vastus lateralis (Fig. 1F). Subsequently, the muscle fibres of the TFL appeared to be inserted into a thin covering fascia of the vastus lateralis muscle (Fig. 2G). The lower extension of the gluteus fascia was still distant from and on the medial side of the initial TFL (Fig. 2A). 

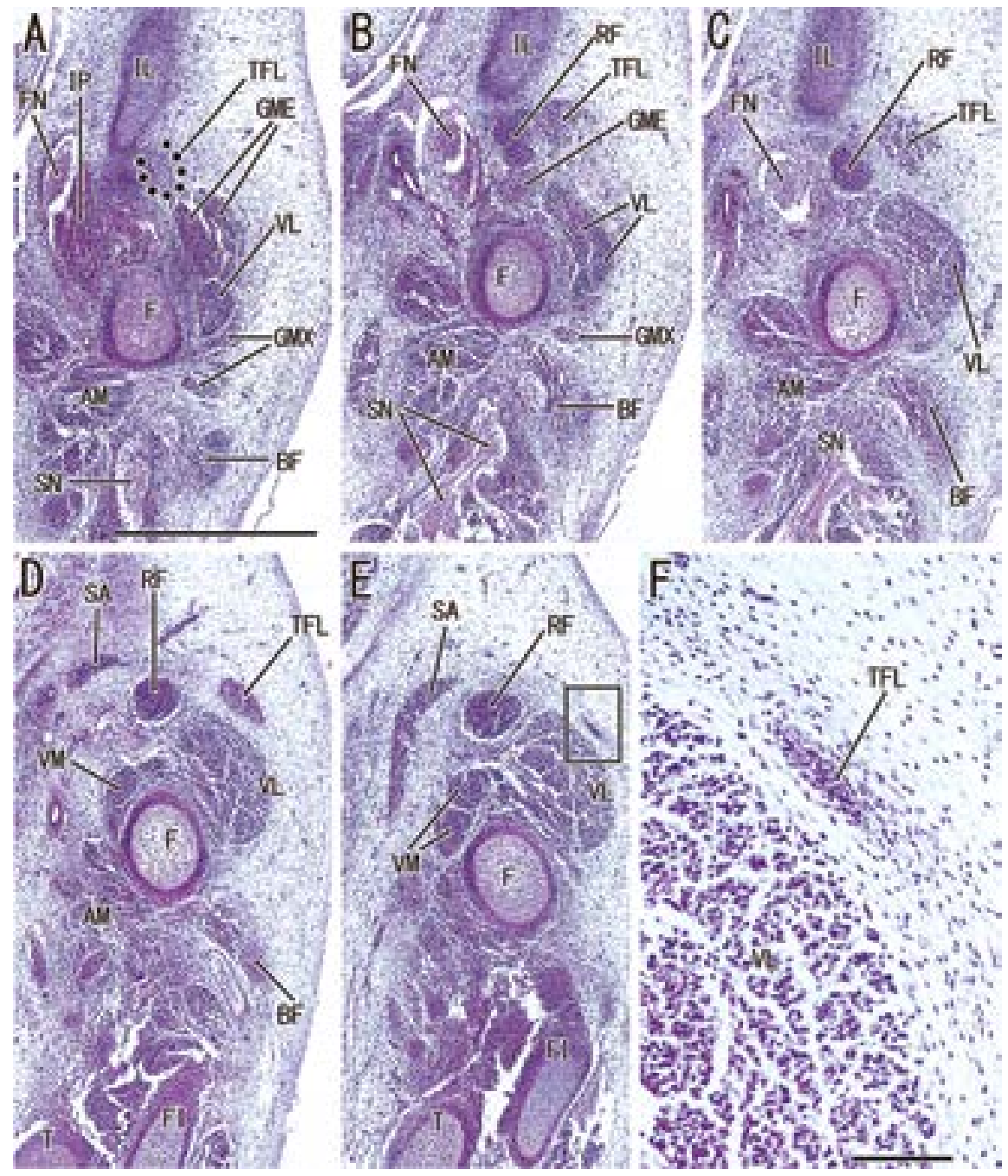

Figure 1. Initial phase of the tensor fasciae latae muscle without definite origin and insertion. Transverse or tilted frontal sections of the thigh. A specimen at 7 weeks (CRL $25 \mathrm{~mm}$ ). H\&E staining. Panel $\mathbf{A}$ or $\mathbf{E}$ displays the most proximal and anterior site (or the most distal and posterior site) in the figure. Panels $\mathbf{A}-\mathbf{E}$ were prepared at the same magnification. Panel $\mathbf{F}$ is a higher magnification view of a square in panel $\mathbf{E}$. Intervals between panels are $0.1 \mathrm{~mm}(A-B, B-C)$ and $0.2 \mathrm{~mm}(C-D, D-E)$, respectively. In panels $\mathbf{A}$ and $\mathbf{B}$, the tensor fascia latae muscle (TFL) appears near the ilium (IL) in the immediately lateral side of the origin of the rectus femoris muscle (RF). The muscle ends as a tendon-like tissue mass on the lateral aspect of the vastus lateralis muscle (VL; panels $\mathrm{E}$ and F) after a short course in subcutaneous tissue. Scale bars: $1 \mathrm{~mm}$ in panel $\mathrm{A}$ and $0.1 \mathrm{~mm}$ in panel $\mathrm{F}$. Common abbreviations for figures: $\mathrm{AM}$ - adductor magnus muscle; $\mathrm{BF}$ - biceps femoris muscle; $\mathrm{CRL}$ — crown-rump length; $\mathrm{F}$ - femur; $\mathrm{FI}$ - fibula; FN — femoral nerve; GME — gluteus medius muscle; GMX — gluteus maximus muscle; H\&E — haematoxylin and eosin; IL — ilium; IP — iliopsoas muscle; RF — rectus femoris muscle; SA — sartorius muscle; SN — sciatic nerve; T — tibia; TFL — tensor fasciae latae muscle; VL — vastus lateralis muscle.

At this stage, the gluteus medius muscle was adjacent to the lateral side of the gluteus maximus.

\section{Observations of larger specimens}

At GA 10-14 weeks (Figs. 3-5), the vastus lateralis fascia or the initial fascia lata was thick and enclosed the entire muscle. However, the vastus fascia turned to the deep side at the posterior intermuscular septum to approach the femur (Figs. 3E; 4B). Thus, the definite fascia lata surrounding the entire thigh muscles was not yet established. At the intermuscular septum, the subcutaneous tissue extended deeply to reach the sciatic nerve. Likewise, the vastus fascia did not reach the tibia (Fig. 4E) but ended at the patella after merging with the lateral margin of the quadriceps tendon (Fig. 5A, B). In contrast to the early morphology (Fig. 2F), the vastus fascia connected to the TFL was separated from the muscle surface by a narrow space (Figs. 3E; 4D; 5C). Moreover, another, thin fascia was detected beneath the vastus fascia (Fig. 4C, D). Simultaneously, a new fascia was developing on the surface of the vastus lateralis muscle.

A small part of the TFL was found to originate from the iliacus (Fig. 4E), whereas most of the muscle 


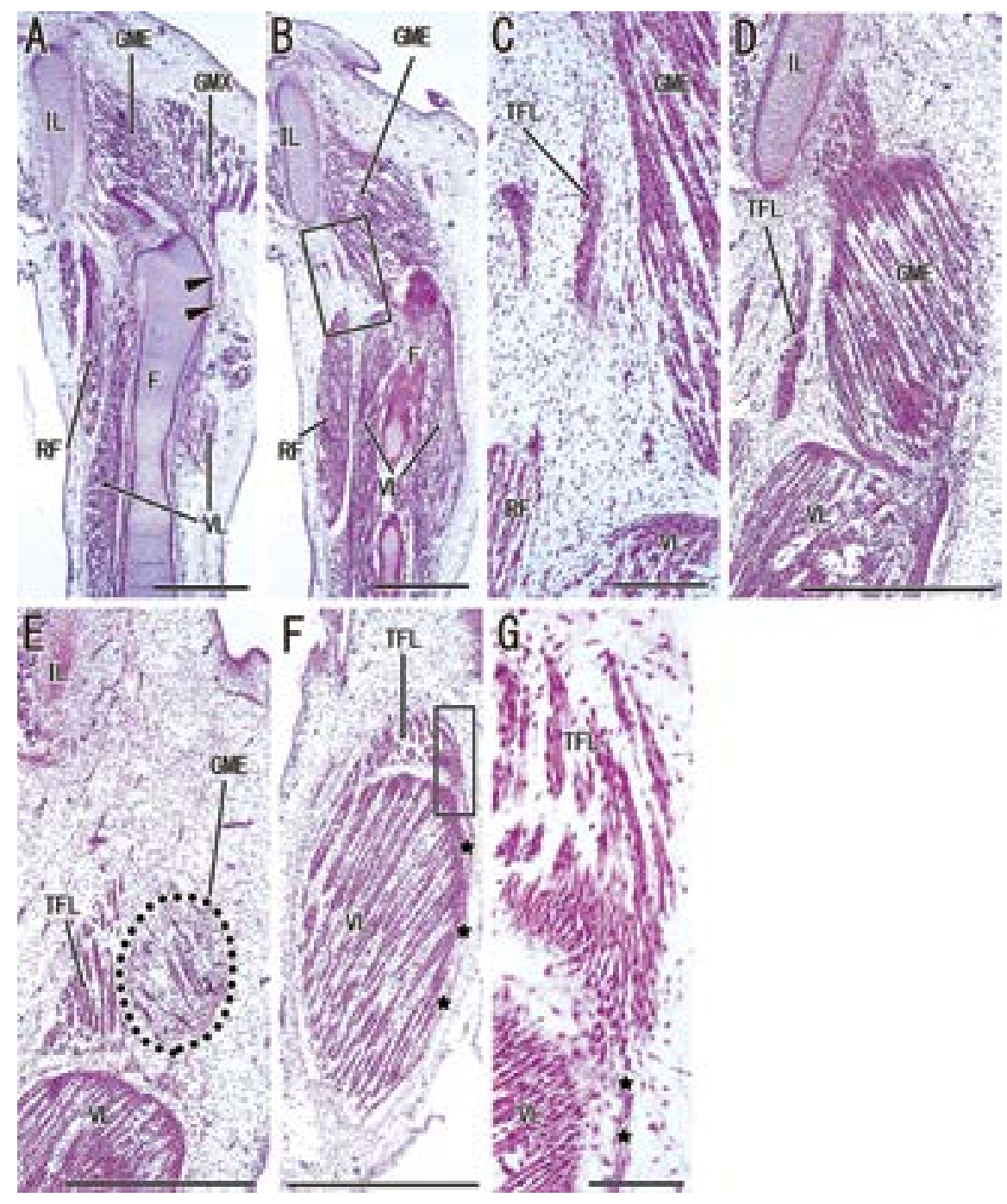

Figure 2. Initial insertion of the tensor fasciae latae muscle. Sagittal sections of the thigh. A specimen at 8 weeks (CRL $35 \mathrm{~mm}$ ). H\&E staining. Panel $\mathbf{A}$ or $\mathbf{F}$ displays the most medial site (or the most lateral site) in the figure. Panels $\mathbf{C}$ or $\mathbf{G}$ is a higher magnification views of a square in panel $\mathbf{B}$ or $\mathbf{F}$. Intervals between panels are $0.4 \mathrm{~mm}(A-B), 0.1 \mathrm{~mm}(B-D)$ and $0.2 \mathrm{~mm}(D-E, E-F)$, respectively. Panel $\mathbf{A}$ exhibits a distal fascial extension (arrowheads) from the gluteus maximus muscle (GMX). The tensor fascia latae muscle (TFL) appears to originate from the ilium (IL in panel D) near the medial margin of the gluteus medius muscle (GME) and inserts into the vastus lateralis fascia covering the vastus lateralis muscle (VL in panels $\mathbf{F}$ and $\mathbf{G}$ ). Scale bars: $1 \mathrm{~mm}$ in panels $A, B, D-F$ and $0.1 \mathrm{~mm}$ in panels $\mathrm{C}$ and $\mathrm{G}$. Other abbreviations - see the common abbreviations in Figure 1.

fibres originated from the gluteus fascia at the inferior or distal end of the gluteus maximus and medius muscles (Figs. 3D, E; 4D; 5D, E). The insertion was found in the superior end of the vastus lateralis fascia. Conversely, the vastus fascia appeared to originate from the inferior end of the TFL (Figs. 3E, F; 4D, F). Subsequently, the TFL became embedded in the vastus fascia because the two laminae of the fascia sandwiched the TFL (Fig. 5C). These two laminae were not always seen in later or larger specimens examined. Thus, they were not yet evident in a specimen of CRL $78 \mathrm{~mm}$ (Fig. 4), despite being present in a specimen of CRL 76 mm (Fig. 5). Subsequently, the gluteus maximus fascia connected to the vastus fascia by way of the fascial lamina superficial to the TFL. Until GA 14 weeks, the TFL connected the gluteus fascia to the patella via the thick, vastus fascia, resulting in an early morphology of the iliotibial tract. However, the final insertion into the tibia was not seen in all specimens examined.

\section{DISCUSSION AND CONCLUSIONS}

We previously considered an initial morphology of the iliotibial tract as an inferior or distal extension of the gluteus fascia [13]. Although our current findings were generally consistent with these earlier results, we found that the distal fascial extension was located 

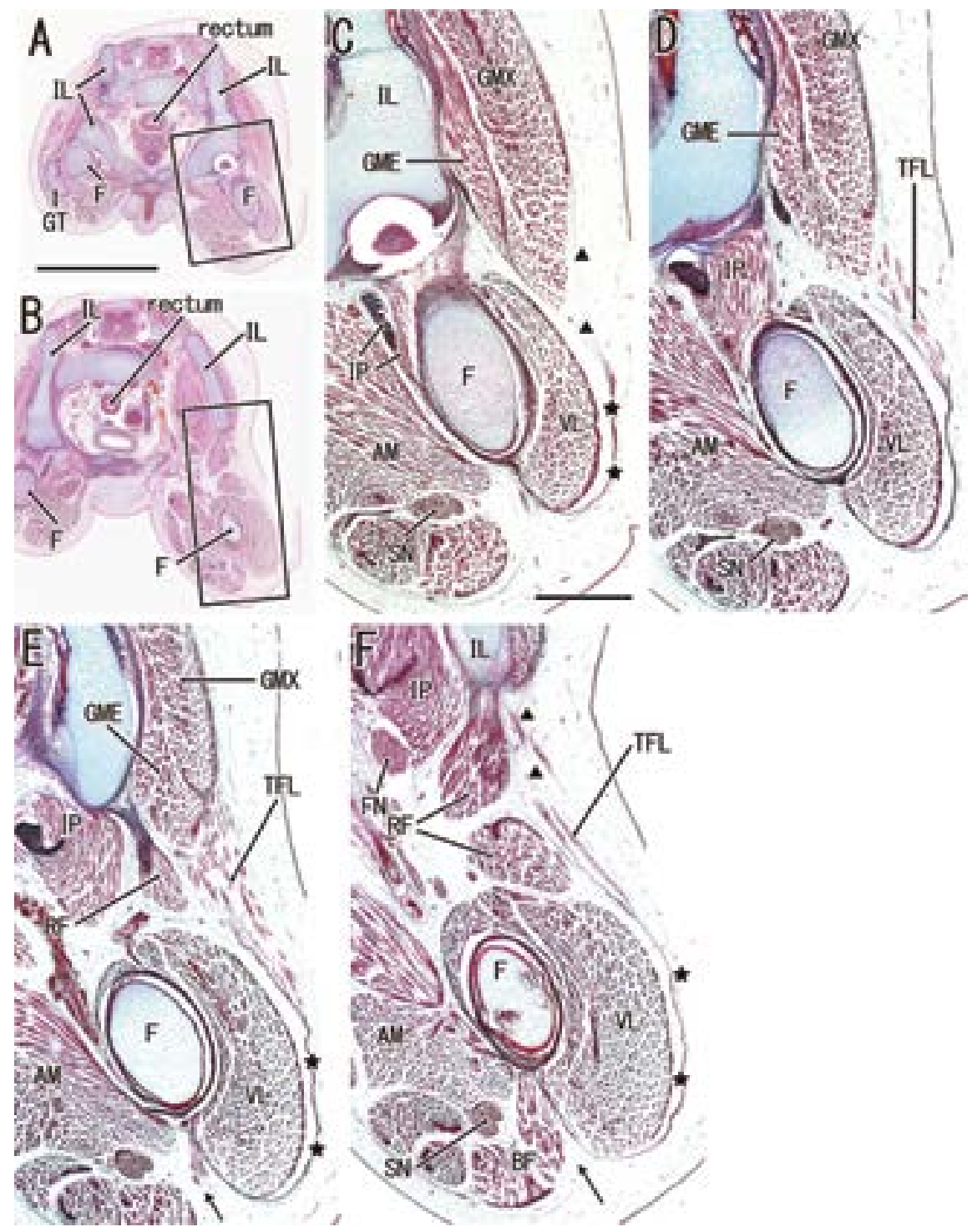

Figure 3. Tensor fascia latae muscle connecting between the gluteus fascia and the vastus lateralis fascia. Horizontal or transverse sections of the thigh. A specimen at 10 weeks (CRL $53 \mathrm{~mm}$ ). H\&E staining. Panels $\mathbf{A}$ and $\mathbf{B}$ exhibit topographical anatomy of the thigh muscles at the lower magnification. Panel $\mathbf{C}$ or $\mathbf{F}$ is a higher magnification view of a square in panel $\mathbf{A}$ or $\mathbf{B}$. Panels $\mathbf{C}-\mathbf{F}$ were prepared at the same magnification. Panel $\mathbf{C}$ or $\mathbf{F}$ displays the most anterior site (or the most posterior site) in the figure. Intervals between panels are $0.2 \mathrm{~mm}(C-D, D-E$, $\mathrm{E}-\mathrm{F})$, respectively. The tensor fasciae latae muscle (TFL) connects between the gluteus muscles (GMX, GME) to the vastus lateralis fascia (asterisk) covering the vastus laterals muscle (VL). A connection between the gluteus and vastus fasciae is still unclear (triangles). At the posterior intermuscular septum (arrow in panels $E$ and F), the vastus fascia turns to the deep side to approach the femur. Scale bars: $5 \mathrm{~mm}$ in panels $\mathrm{A}$ and $\mathrm{E} ; 1 \mathrm{~mm}$ in panel B. Other abbreviations — see the common abbreviations in Figure 1.

on the posterolateral side of the developing TFL. Thus, the TFL did not attach to the fascial extension but to the inferomedial margin of the gluteus maximus and medius muscles. Therefore, our candidate for the initial iliotibial tract (i.e., the gluteus fascial extension) may have a more limited role than previously thought. This study found that a fascia covering the gluteus maximus rather than the distal extension was connected to the vastus lateralis fascia by the TFL. The connecting fascia divided into two laminae to sandwich the TFL in the larger specimens, a morphology similar to that of muscle embedded in the iliotibial tract in adults. However, in the present specimens, the early tract did not reach the tibia but merged with the quadriceps tendon. The final approach of the tract to the tibia likely required maturation of the vastus fascia, making them parts of the future fascia lata. This structure would become multilayered and separate from the quadriceps tendon, possibly after birth (see also the third paragraph).

In adults, the TFL is not a major actor but provides spaces for other muscle functions. The most 


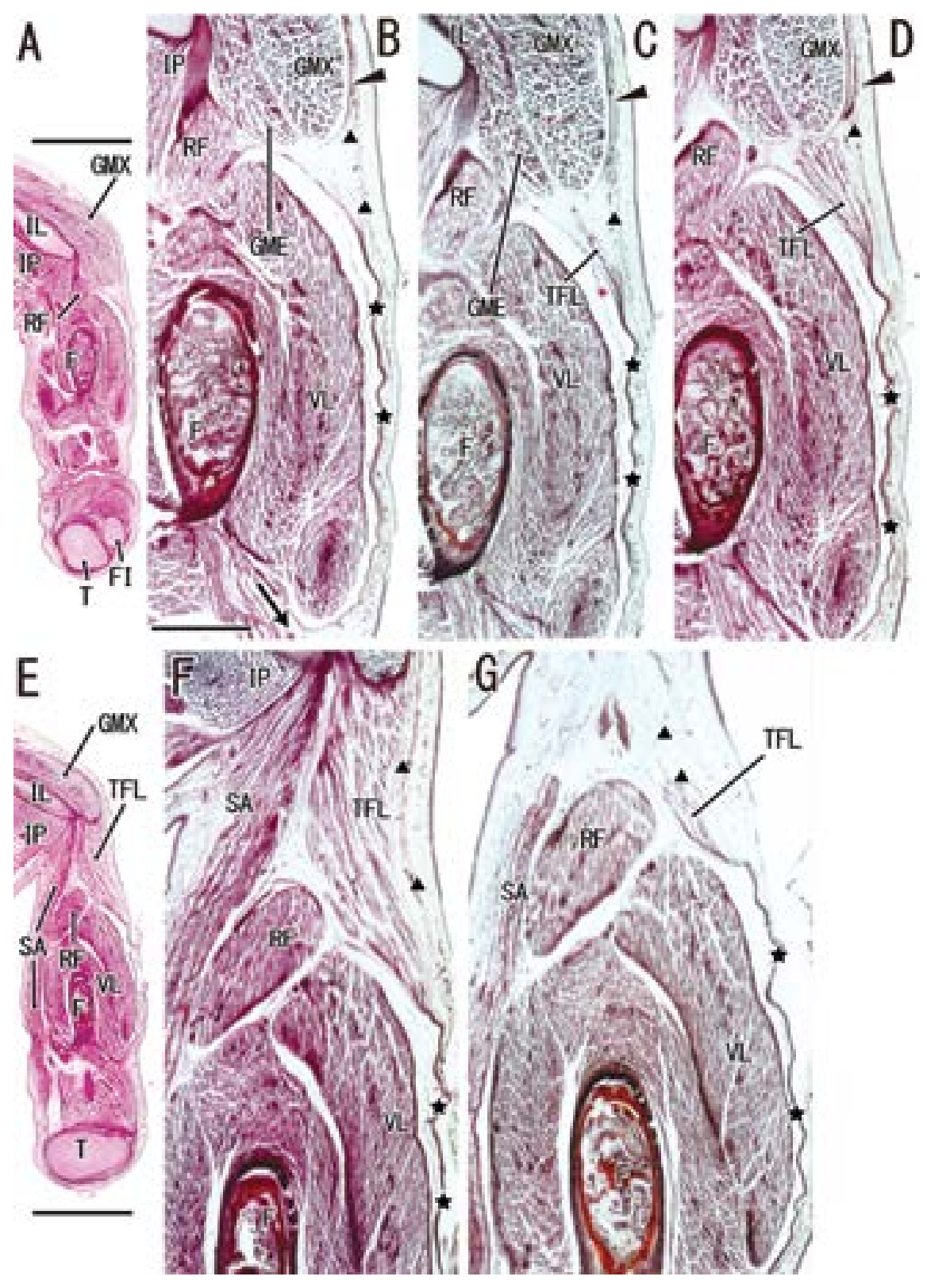

Figure 4. Gluteus fascia connecting to the vastus lateralis fascia on the tensor fasciae latae muscle. Tilted frontal sections of the thigh. A specimen at 12 weeks (CRL $78 \mathrm{~mm}$ ). H\&E staining. Panels $\mathbf{A}$ and $\mathbf{E}$ exhibit topographical anatomy of the thigh muscles at the lower magnification. Panel $\mathbf{B}$ or $\mathbf{F}$ corresponds to the central part of panel $\mathbf{A}$ or $\mathbf{E}$. Panels B-D, $\mathbf{F}$, and $\mathbf{G}$ were prepared at the same magnification. Panel $\mathbf{B}$ or $\mathbf{G}$ displays the most anterior site (or the most posterior site) in the figure. Intervals between panels are $0.1 \mathrm{~mm}(B-C, C-D), 0.8 \mathrm{~mm}(D-E)$ and $0.3 \mathrm{~mm}(\mathrm{E}-\mathrm{G})$, respectively. Panels $\mathbf{B}$ and $\mathbf{C}$ exhibit the vastus lateralis fascia (asterisk) and the gluteus fascia (arrowhead). A connection of these fasciae is now developing (triangles). At the posterior intermuscular septum (arrow in panel B), the vastus fascia turns to the deep side to approach the femur. Thus, the fascia does not reach the tibia (panel E). Scale bars: $5 \mathrm{~mm}$ in panels A and E; $1 \mathrm{~mm}$ in panel B. Other abbreviations - see the common abbreviations in Figure 1.

effective action of the TFL is highly dependent on hip joint position and femoral neck angle $[5,6,14]$. TFL function is abolished by nerve injury during hip surgery, but its function can be compensated by the other hip muscles after rehabilitation $[2,10]$. However in contrast to the limited muscle function in adults, the foetal TFL plays a role in connecting the gluteus fascia to the vastus fascia. In the absence of this connection, the activity of the gluteus maximus muscle would be limited to abduction and flexion at the hip joint, as in quadrupedal mammals. The evolutionary transition from quadripedality to bipedality and a permanently upright posture require not only the iliotibial tract but also its strong upward traction by the gluteus maximus. In foetuses, rather than the iliac crest, the TFL originated from not 


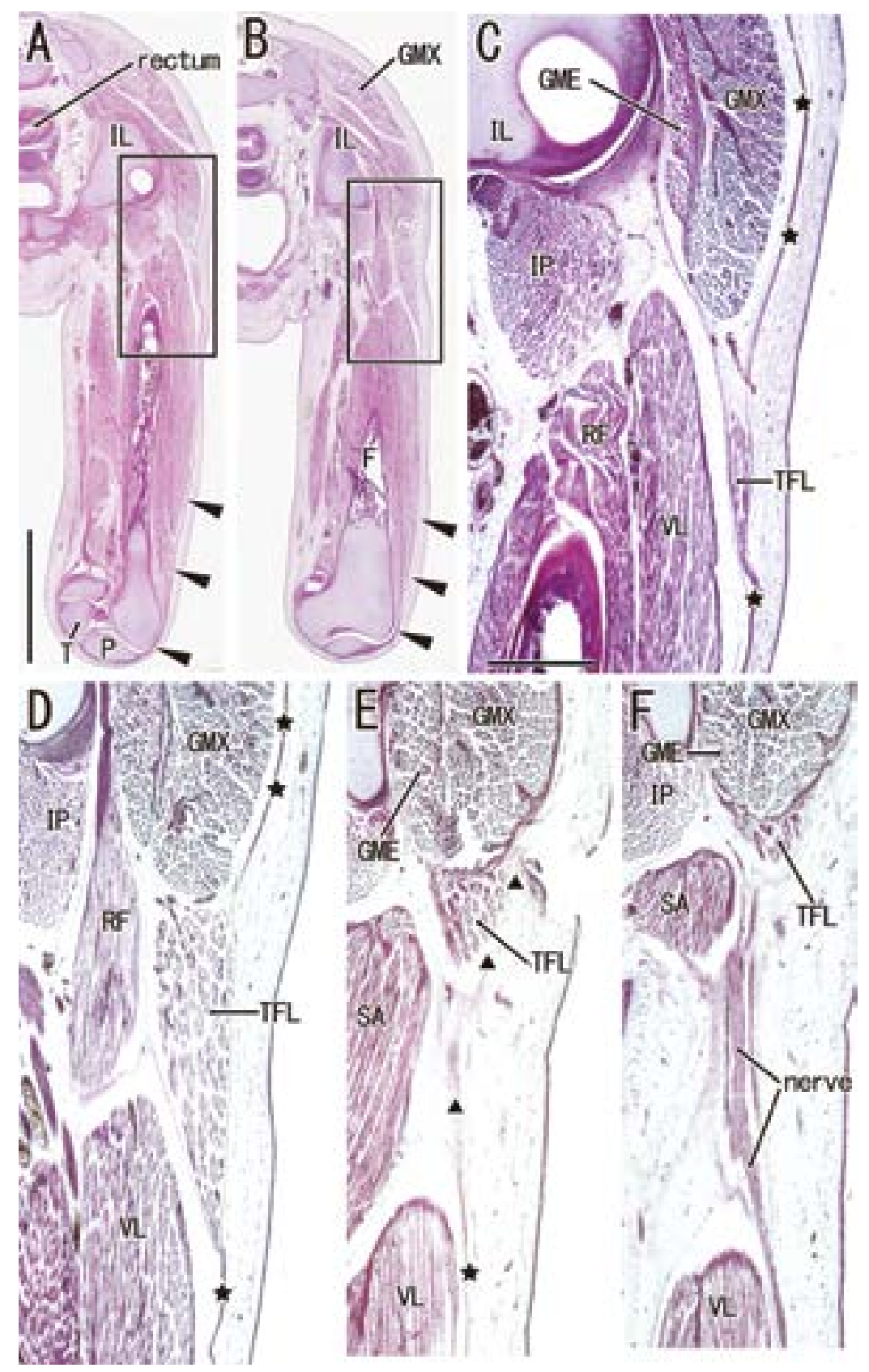

Figure 5. Tensor fasciae latae muscle sandwiched by two laminae of the early iliotibial tract. Longitudinal and frontal sections of the thigh. A specimen at 12 weeks (CRL $76 \mathrm{~mm}$ ). H\&E staining. Panels $\mathbf{A}$ and $\mathbf{B}$ exhibit topographical anatomy of the thigh muscles at the lower magnification. Panel $\mathbf{C}$ or $\mathbf{D}$ is a higher magnification view of a square in panel $\mathbf{A}$ or $\mathbf{B}$. Panels $\mathbf{C}-\mathbf{F}$ were prepared at the same magnification. Panel $\mathbf{C}$ or $\mathbf{F}$ displays the most anterior site (or the most posterior site) in the figure. Intervals between panels are $0.4 \mathrm{~mm}(\mathrm{C}-\mathrm{D}), 0.8 \mathrm{~mm}(\mathrm{D}-\mathrm{E})$ and $0.2 \mathrm{~mm}(\mathrm{E}-\mathrm{F})$, respectively. Panel $\mathbf{C}$ exhibits two laminae of the early iliotibial tract (black asterisk, the superficial lamina) sandwiching the tensor fasciae latae muscle (TFL). The muscle does not attach to the ilium (IL) but to the gluteus muscles (GMX, GME). The early iliotibial tract ends at the patella (arrowheads in panels $\mathbf{A}$ and $\mathbf{B}$ ), but it disappears in the posterior sites (triangles in panels $\mathbf{E}$ and $\mathbf{F}$ ). A nerve in panel $\mathbf{F}$ is the posterior cutaneous nerve of thigh. Scale bars: $5 \mathrm{~mm}$ in panel $A ; 1 \mathrm{~mm}$ in panel C. Other abbreviations — see the common abbreviations in Figure 1.

only the gluteus maximus but the gluteus medius. A mechanical requirement for standing and walking after birth as well as prenatal internal rotation of the femur may result in functional differentiation between the gluteus medius and maximus muscles, resulting in the TFL more tightly connecting with the gluteus maximus.

The vastus lateralis fascia became separated from the muscle surface by a narrow space. This separation was apparently required for iliotibial tract function in- 
dependent of the quadriceps tendon. Foetal development of the fascia lata is not simple. The multi-layered configuration first originates from the surface of the vastus muscles, with the first or outermost layer originating and being stripped from the muscle surface in association with the development of the second layer, with this procedure repeating until three or more layers are stripped [7]. Thus, the TFL connected the outermost fascia with the gluteus fascia. As the third or fourth layer of the fascia lata is added, possibly after birth, the initial patellar attachment of the iliotibial tract may be loosened or interrupted, accompanied by simultaneous final insertion of the tract into the tibia. Finally, traction by the biceps femoris muscle is thought to drive production of the distal extension of the gluteus fascia [13]. However, rather than the biceps, a growing greater trochanter of the femur may compel the fascia to extend more distally and laterally.

Finally, there were two limitations in this study. 1) We did not have any information about cause of death of the specimens. Thus, we did not eliminate a possibility that some anomalies were included in the anatomy described. 2) Even the latest or largest specimens did not show the final morphology of the iliotibial tract. Therefore, a further study was necessary to ensure our hypothesis.

\section{Acknowledgements}

We are grateful to Mr. H.G. Sydow for his assistance with the Blechschmidt collection, Göttingen. This study was supported in part by a Grant-in-Aid for Scientific Research (JSPS KAKENHI No. 16K08435) from the Ministry of Education, Culture, Sports, Science and Technology in Japan.

\section{REFERENCES}

1. Al-Hayani A. The functional anatomy of hip abductors. Folia Morphol. 2009; 68(2): 98-103, indexed in Pubmed: 19449297.

2. Apaydin N, Kendir S, Loukas M, et al. Surgical anatomy of the superior gluteal nerve and landmarks for its localization during minimally invasive approaches to the hip. Clin Anat. 2013; 26(5): 614-620, doi: 10.1002/ca.22057, indexed in Pubmed: 22374811.

3. Baczkowski $K$, Marks $P$, Silberstein $M$, et al. A new look into kicking a football: an investigation of muscle activity using MRI. Australas Radiol. 2006; 50(4): 324-329, doi: 10.1111/j.1440-1673.2006.01591.x, indexed in Pubmed: 16884417.

4. Bardeen RC. Development and variation of the musculature of the inferior extremity and of the neighboring regions of the trunk in man. Am J Anat. 1907; 6: 332-336.

5. Birnbaum K, Siebert $\mathrm{CH}$, Pandorf T, et al. Anatomical and biomechanical investigations of the iliotibial tract. Surg Radiol Anat. 2004; 26(6): 433-446, doi: 10.1007/s00276004-0265-8, indexed in Pubmed: 15378277.

6. Casartelli NC, Leunig M, Item-Glatthorn JF, et al. Hip muscle weakness in patients with symptomatic femoroacetabular impingement. Osteoarthritis Cartilage. 2011; 19(7): 816-821, doi: 10.1016/j.joca.2011.04.001, indexed in Pubmed: 21515390.

7. Cho BH, Kim JiH, Jin ZWu, et al. Topographical anatomy of the intestines during in utero physiological herniation. Clin Anat. 2018; 31(4): 583-592, doi: 10.1002/ca.22996, indexed in Pubmed: 29044646.

8. Fu SN, Hui-Chan CWY. Modulation of prelanding lower-limb muscle responses in athletes with multiple ankle sprains. Med Sci Sports Exerc. 2007; 39(10): 1774-1783, doi: 10.1249/mss.0b013e3181343629, indexed in Pubmed: 17909405.

9. Gottschalk F, Kourosh S, Leveau B. The functional anatomy of tensor fasciae latae and gluteus medius and minimus. J Anat. 1989; 166: 179-189, indexed in Pubmed: 2621137.

10. Grob K, Manestar M, Ackland T, et al. Potential risk to the superior gluteal nerve during the anterior approach to the hip joint: an anatomical study. J Bone Joint Surg Am. 2015; 97(17): 1426-1431, doi: 10.2106/JBJS.0.00146, indexed in Pubmed: 26333738.

11. Huang BK, Campos JC, Michael Peschka PG, et al. Injury of the gluteal aponeurotic fascia and proximal iliotibial band: anatomy, pathologic conditions, and MR imaging. Radiographics. 2013; 33(5): 1437-1452, doi: 10.1148/ rg.335125171, indexed in Pubmed: 24025934.

12. Paré EB, Stern JT, Schwartz JM. Functional differentiation within the tensor fasciae latae. A telemetered electromyographic analysis of its locomotor roles. J Bone Joint Surg Am. 1981; 63(9): 1457-1471, indexed in Pubmed: 7320037.

13. Shiraishi Y, Jin ZW, Mitomo K, et al. Foetal development of the human gluteus maximus muscle with special reference to its fascial insertion. Folia Morphol. 2018; 77(1): 144-150, doi: 10.5603/FM.a2017.0060, indexed in Pubmed: 28653302.

14. Sobczak S, Dugailly PM, Feipel V, et al. In vitro biomechanical study of femoral torsion disorders: effect on moment arms of thigh muscles. Clin Biomech (Bristol, Avon). 2013; 28(2): 187-192, doi: 10.1016/j.clinbiomech.2012.12.008, indexed in Pubmed: 23337767.

15. Sutter R, Kalberer F, Binkert CA, et al. Abductor tendon tears are associated with hypertrophy of the tensor fasciae latae muscle. Skeletal Radiol. 2013; 42(5): 627-633, doi: 10.1007/ s00256-012-1514-2, indexed in Pubmed: 22940837. 